\title{
Persistent Current in Metallic Rings and Cylinders
}

\author{
Santanu K. Maiti \\ E-mail: santanu.maiti@saha.ac.in \\ ${ }^{1}$ Theoretical Condensed Matter Physics Division \\ Saha Institute of Nuclear Physics \\ 1/AF, Bidhannagar, Kolkata-700 064, India \\ ${ }^{2}$ Department of Physics \\ Narasinha Dutt College \\ 129, Belilious Road, Howrah-711 101, India
}




\section{Contents}

Preface

1 Introduction $\quad 4$

2 Persistent Current in Non-Interacting Single-Channel and MultiChannel Mesoscopic Rings

2.1 Origin of Persistent Current . . . . . . . . . . . . . . 5

2.2 Non-Interacting One-Channel Rings . . . . . . . . . . . . . . . 7

2.2.1 Impurity Free Rings . . . . . . . . . . . . . . . . . 7

2.2 .2 Rings with Impurity . . . . . . . . . . . . . . . . 9

2.3 Non-Interacting Multi-Channel Systems . . . . . . . . . . . . . . . 11

2.3.1 Energy Spectra . . . . . . . . . . . . . . 13

2.3.2 Persistent Current ........................ 14

3 Low-Field Magnetic Response on Persistent Current 18

3.1 One-Channel Mesoscopic Rings . . . . . . . . . . . . . . . . . . 19

3.1.1 Effect of Temperature ..................... 20

3.2 Multi-Channel Mesoscopic Cylinders ................ . . 21

4 Concluding Remarks $\quad 22$

\begin{tabular}{ll|} 
References & 23 \\
\hline
\end{tabular} 


\section{Preface}

We explore the behavior of persistent current and low-field magnetic response in mesoscopic one-channel rings and multi-channel cylinders within the tight-binding framework. We show that the characteristic properties of persistent current strongly depend on total number of electrons $N_{e}$, chemical potential $\mu$, randomness and total number of channels. The study of low-field magnetic response reveals that only for one-channel rings with fixed $N_{e}$, sign of the low-field currents can be predicted exactly, even in the presence of disorder. On the other hand, for multi-channel cylinders, sign of the low-field currents cannot be mentioned exactly, even in the perfect systems with fixed $N_{e}$ as it significantly depends on the choices of $N_{e}, \mu$, number of channels, disordered configurations, etc.

Keywords: Mesoscopic ring; Mesoscopic cylinder; Persistent current; Magnetic susceptibility. 


\section{Introduction}

The physics of small metallic rings provides an excellent testing ground for many ideas of basic physics. In thermodynamic equilibrium, a small metallic ring threaded by a magnetic flux $\phi$ supports a current that does not decay dissipatively even at non-zero temperature. It is the so-called persistent current in mesoscopic normal metal rings. This phenomenon is a purely quantum mechanical effect and gives an obvious demonstration of the Aharonov-Bohm effect [1]. The possibility of persistent current was predicted in the very early days of quantum mechanics by Hund [2], but their experimental evidences came much later only after realization of the mesoscopic systems. In 1983, Büttiker et al. [3] predicted that persistent current can exist in mesoscopic normal metal rings threaded by a magnetic flux even in the presence of impurity. Few years later, in a pioneering experiment Levy et al. [4 first gave the experimental evidence of persistent current in mesoscopic metallic rings. Following with this, the existence of the persistent current was further confirmed by several experiments [5, 6, 7, 8, 9, 10]. Though there exists a vast literature of theoretical [11, 12, 13, 14, 15, 16, 17, 18, 19, 20, 21, 22, 23, 24, 26, 27, 28, 29, 30, 31] as well as experimental [4, 5, 6, 7, 8, 9, 10] results on persistent currents, but lot of controversies are still present between the theory and experiment. For our illustrations, here we mention very briefly some of them as follow. (i) The main controversy appears in the determination of the current amplitude. It has been observed that the measured current amplitude exceeds an order of magnitude than the theoretical estimates. Many efforts have been paid to solve this problem, but no such proper explanation has yet been found out. Since normal metals are intrinsically disordered, it was believed that electronelectron correlation can enhance the current amplitude by homogenize the system [32]. But the inclusion of the electron correlation [33] doesn't give any significant enhancement of the persistent current. Later, in some recent papers [34, 35, 36] it has been studied that the simplest nearest-neighbor tight-binding model with electron-electron interaction cannot explain the actual mechanisms. The higher order hopping integrals in addition to the nearest-neighbor hopping integral have an important role to magnify the current amplitude in a considerable amount. With this prediction some discrepancies can be removed, but the complete mechanisms have to be understood. (ii) The appearance of different flux-quantum periodicities rather than simple $\phi_{0}\left(\phi_{0}=c h / e\right.$, the elementary flux-quantum) periodicity in persistent current is not quite clear to us. The presence of other flux-quantum periodicities has already been reported in many papers [37, 38, 39, 40, but still there exist so many conflict. (iii) The prediction of the sign of low-field currents is a major challenge in this area. Only for a single-channel ring, the sign of the low-field 
currents can be mentioned exactly [40, 41]. While, in all other cases i.e., for multi-channel rings and cylinders, the sign of the low-field currents cannot be predicted properly. It then depends on the total number of electrons $\left(N_{e}\right)$, chemical potential $(\mu)$, disordered configurations, etc. Beside these, there are several other controversies those are unsolved even today. Thus it can be emphasized that the study of persistent current in normal metal rings, cylinders and other loop geometries is an open challenge to us. In the present article we investigate the behavior of persistent current in mesoscopic rings and cylinders within the non-interacting electron picture. This study may be helpful for the beginners to understand the basic features of persistent current in metallic loops.

The article is organized as follow. In Section 2, we illustrate the behavior of persistent current in mesoscopic normal metal rings and cylinders. Section 3 focuses the magnetic response of low-field currents both at absolute zero and finite temperatures. Finally, we conclude our results in Section 4.

\section{Persistent Current in Non-Interacting Single- Channel and Multi-Channel Mesoscopic Rings}

Our aim of this section is to study persistent currents in some small non-superconducting loops threaded by a magnetic flux $\phi$. A conducting ring, penetrated by a magnetic flux $\phi$, carries an equilibrium current in its ground state that persists in time. An electrically charged particle moving around the ring but not entering the region of magnetic flux, feels no (classical) force during its motion. However, the magnetic vector potential $\vec{A}$, related to the magnetic field through the relation $\vec{B}=\vec{\nabla} \times \vec{A}$, affects the quantum state of the particle by changing the phase of its wave function. As a consequence, both thermodynamic and kinetic properties oscillate with the magnetic flux $\phi$. Here we present some analytical as well as numerical calculations and study the behavior of persistent current $I$ in mesoscopic rings as a function of flux $\phi$, system size $L$, total number of electrons $N_{e}$, chemical potential $\mu$, strength of disorder $W$ and total number of channels.

\subsection{Origin of Persistent Current}

In this sub-section we describe how persistent current appears in a small normal metal ring threaded by a magnetic flux $\phi$. The schematic representation of the system is given in Fig. 1. The electric field $\mathcal{E}$ associated with the magnetic field $B$ in the ring can be expressed through the relation (Faraday's law),

$$
\vec{\nabla} \times \overrightarrow{\mathcal{E}}=-\frac{1}{c} \frac{\partial \vec{B}}{\partial t}
$$


Using the above relation we can determine the electric field $\mathcal{E}$ from the following expressions:

$$
\oint_{S}(\vec{\nabla} \times \overrightarrow{\mathcal{E}}) \cdot \overrightarrow{d S}=-\frac{1}{c} \oint_{S} \frac{\partial \vec{B}}{\partial t} \cdot \overrightarrow{d S}=-\frac{1}{c} \frac{\partial}{\partial t} \oint_{S} \vec{B} \cdot \overrightarrow{d S}=-\frac{1}{c} \frac{\partial \phi}{\partial t}
$$

where $S$ is the area enclosed by the ring. From the Stoke's theorem we can write,

$$
\oint_{\text {loop }} \overrightarrow{\mathcal{E}} \cdot \overrightarrow{d l}=-\frac{1}{c} \frac{\partial \phi}{\partial t}
$$

and thus the electric field can be written in terms of the time variation of the magnetic

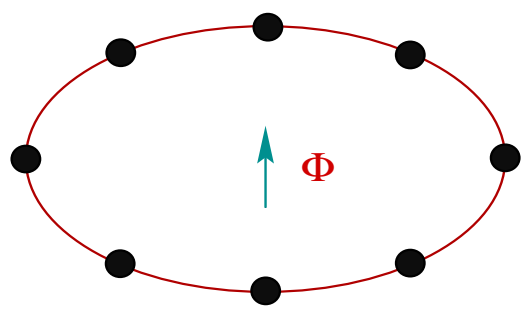

Figure 1: One-dimensional mesoscopic ring threaded by a magnetic flux $\phi$, where the filled circles correspond to the position of the atomic sites. A persistent current $I$ is established in the ring.

flux $\phi$ as,

$$
\mathcal{E}=-\frac{1}{2 \pi r c} \frac{\partial \phi}{\partial t}
$$

where $r$ is the radius of the ring. Therefore, the force acting on an electron in the ring becomes,

$$
F=-\frac{e}{2 \pi r c} \frac{\partial \phi}{\partial t}
$$

and the change in energy or work done for a small displacement $\triangle s$ is,

$$
\triangle E=\triangle W=\vec{F} \cdot \overrightarrow{\triangle s}=-\frac{e}{2 \pi r c} \frac{\triangle \phi}{\triangle t} \triangle s=-\frac{e}{2 \pi r c} \triangle \phi\left(\frac{\triangle s}{\triangle t}\right)
$$

The velocity of the electron in the ring can be expressed as,

$$
v=\frac{\triangle s}{\triangle t}=-\frac{2 \pi r c}{e}\left(\frac{\triangle E}{\triangle \phi}\right)
$$

and the persistent current that developed in the ring becomes,

$$
I=e f=\frac{e v}{2 \pi r}=-c\left(\frac{\triangle E}{\triangle \phi}\right)
$$

This is the final expression of persistent current, and we see that the current carried by an energy eigenstate is obtained by taking the first order derivative of the energy for that particular state with respect to the magnetic flux $\phi$. For the sake of simplicity, throughout our studies we use the units where $c=1, e=1$ and $h=1$. 


\subsection{Non-Interacting One-Channel Rings}

Here we focus our attention on the behavior of persistent currents in one-channel rings [41], where all the currents are computed for the non-interacting electron picture based on the tight-binding formulation. The model Hamiltonian for a $N$-site ring $(L=N a, a$ is the lattice spacing) threaded by a magnetic flux $\phi$ (in units of the elementary flux-quantum $\left.\phi_{0}=c h / e\right)$ can be expressed in this form,

$$
H=\sum_{i} \epsilon_{i} c_{i}^{\dagger} c_{i}+\sum_{<i j>} t\left[e^{i \theta} c_{i}^{\dagger} c_{j}+e^{-i \theta} c_{j}^{\dagger} c_{i}\right]
$$

where $c_{i}^{\dagger}\left(c_{i}\right)$ corresponds to the creation (annihilation) operator of an electron at the site $i, t$ represents the nearest-neighbor hopping strength, $\epsilon_{i}$ 's are the on-site energies and $\theta=2 \pi \phi / N$ is the phase factor due to the flux $\phi$ threaded by the ring. The magnetic flux $\phi$ enters explicitly into the above Hamiltonian (Eq. 9), and the wave functions satisfy the periodic boundary condition which is equivalent to consider the above Hamiltonian at zero flux with the flux-modified boundary conditions:

$$
\begin{aligned}
\left.\psi\right|_{x=L} & =\left.\exp \left[\frac{2 \pi i \phi}{\phi_{0}}\right] \psi\right|_{x=0} \\
\left.\frac{d \psi}{d x}\right|_{x=L} & =\left.\exp \left[\frac{2 \pi i \phi}{\phi_{0}}\right] \frac{d \psi}{d x}\right|_{x=0}
\end{aligned}
$$

here $x$ varies between 0 to $L$ and is expressed as $x=L \theta^{\prime} / 2 \pi$, where $\theta^{\prime}$ is the azimuthal angle, the spatial degrees of freedom of the electron in the ring.

\subsubsection{Impurity Free Rings}

In order to reveal the basic properties of persistent currents, let us begin our discussion with the simplest possible system which is the case of impurity free non-interacting electron model.

\section{Energy Spectra}

For a perfect ring, setting $\epsilon_{i}=0$ for all $i$, we get the energy of $n$th eigenstate as,

$$
E_{n}(\phi)=2 t \cos \left[\frac{2 \pi}{N}\left(n+\frac{\phi}{\phi_{0}}\right)\right]
$$

where $n=0, \pm 1, \pm 2, \ldots$. In Fig. 2, we plot the energy-flux $(E$ - $\phi)$ characteristics for a typical ordered ring considering its size $N=10$. From the spectrum it is observed that the energy levels of the ring vary periodically with $\phi$ showing $\phi_{0}$ flux-quantum periodicity. At an integer or half-integer flux quantum, the energy levels have an extrema i.e., either a 


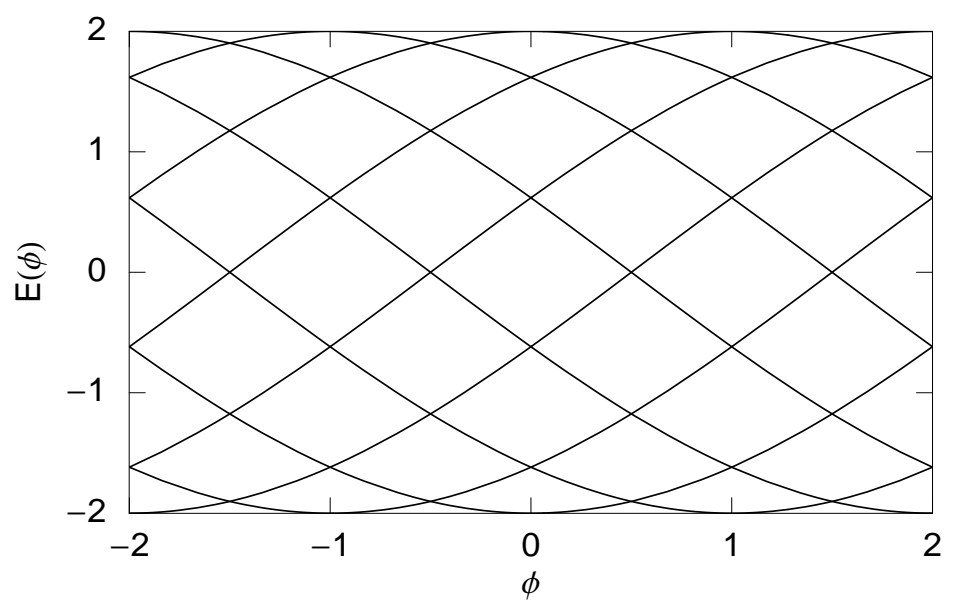

Figure 2: Electron energy levels as a function of the magnetic flux $\phi$ for a one-dimensional impurity-free ring considering the system size $N=10$.

maximum or a minimum, and accordingly, at these values of $\phi$ persistent current should vanish since it is computed from the first order derivative of the energy eigenvalue. In the following, we discuss the current-flux $(I-\phi)$ characteristics for the two different cases. For one case we consider the rings with fixed number of electrons $N_{e}$, while for the other case the rings have some fixed chemical potential $\mu$.

\section{Persistent Current: Rings with Fixed $N_{e}$}

The current carried by the $n$th energy eigenstate, whose energy is given by Eq. 11, can be obtained through the expression,

$$
I_{n}(\phi)=\left(\frac{4 \pi t}{N \phi_{0}}\right) \sin \left[\frac{2 \pi}{N}\left(n+\frac{\phi}{\phi_{0}}\right)\right]
$$

At absolute zero temperature $(T=0 \mathrm{~K})$, the total persistent current is obtained by taking the sum of individual contributions from the lowest $N_{e}$ energy eigenstates. For our illustrative purpose, in Fig. 3, we display the current-flux characteristics for some typical one-channel perfect rings $(N=50)$, where (a) corresponds to the results for the rings with odd $N_{e}$ and (b) represents the results for the rings with even $N_{e}$. The results predict that the current shows saw-tooth like nature with sharp transitions at half-integer and integer flux quanta for the rings with odd and even $N_{e}$ respectively. For all such cases, the current varies periodically with $\phi$ providing $\phi_{0}$ flux-quantum periodicity.

\section{Persistent Current: Rings with Fixed $\mu$}

For the rings described with fixed chemical potential $\mu$, instead of $N_{e}$, the total persistent current at $T=0 \mathrm{~K}$ will be obtained by adding all the individual contributions from the 

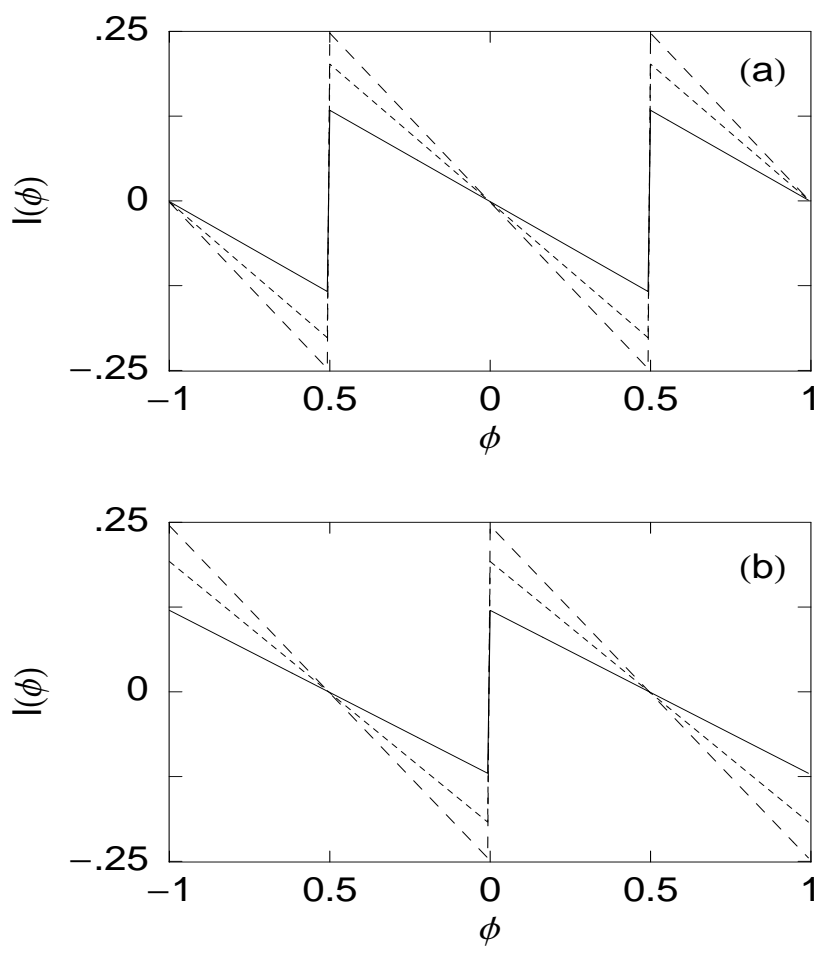

Figure 3: Current-flux characteristics for some one-channel perfect rings $(N=50)$ described with fixed number of electrons. The solid, dotted and dashed curves in (a) correspond to $N_{e}=9,15$ and 23 electrons respectively, while in (b) these curves correspond to $N_{e}=8,14$ and 22 electrons respectively.

energy levels with energies less than or equal to $\mu$. As the chemical potential is fixed, the total number of electrons varies as a function of the magnetic flux $\phi$ except for some special choices of $\mu$, where the rings contain fixed number of electrons. In Fig. 4, we plot the persistent currents for some one-channel perfect rings those are described with fixed chemical potential $\mu$. Here we take the ring size $N=100$. Figures 4(a) and (b) correspond to the persistent currents for the rings with $\mu=-1$ and -1.5 respectively. Our results predict that several additional kink-like structures appear at different field points and their positions also depend on the choices of $\mu$. For all these cases the current gets only $\phi_{0}$ periodicity, as expected.

\subsubsection{Rings with Impurity}

Metals are intrinsically disordered which tends to decrease persistent current due to the localization effect [42] of energy eigenstates. In order to emphasize the role of impurities on persistent currents now we concentrate our study on the rings in the presence of disorder. 

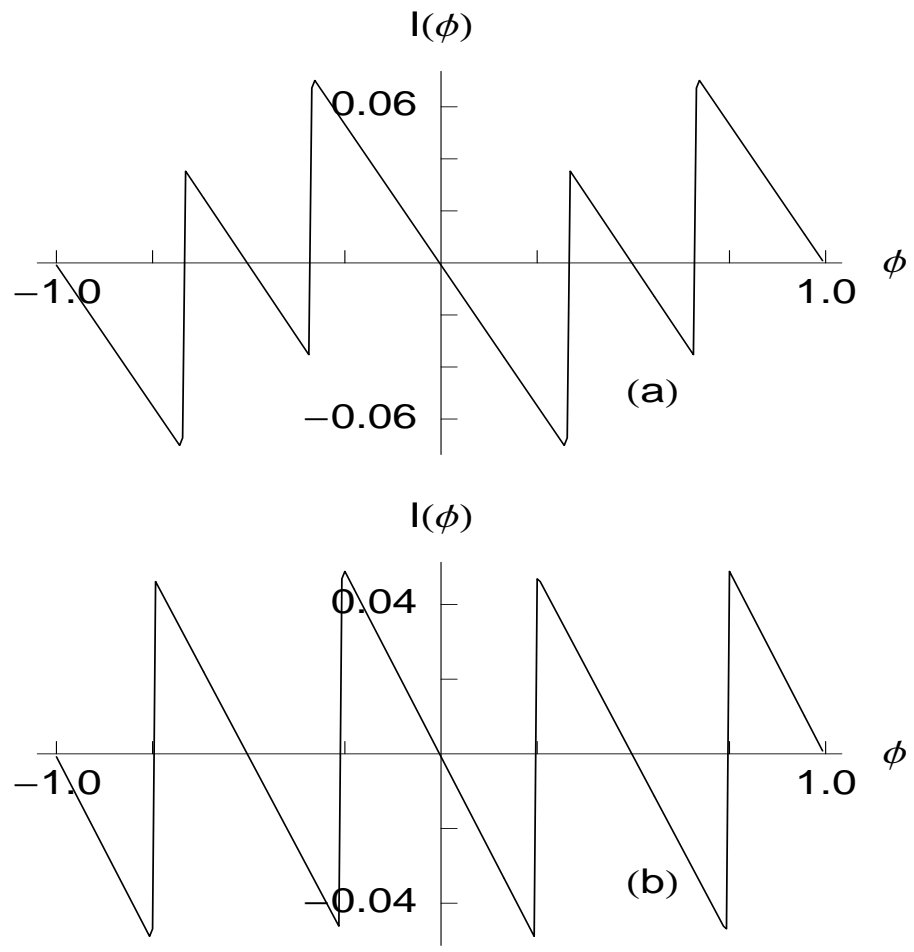

Figure 4: Current-flux characteristics for the one-channel perfect rings $(N=100)$ described with constant $\mu$, where (a) $\mu=-1$ and (b) $\mu=-1.25$.

To introduce the impurities in the ring, we choose the site energies $\epsilon_{i}$ 's randomly from a "Box" distribution function of width $W=1$, which reveal that the ring is subjected to the diagonal disorder. In the presence of impurity in the ring, gaps open at the points of intersection of the energy levels, in the same way as band gaps form in the bandstructure problem, and they vary continuously with the magnetic flux $\phi$. Figure 5 shows the variation of the energy eigenstates as a function of $\phi$ for a one-channel ring $(N=10)$ in the presence of diagonal disorder. The continuous variation of the energy levels with respect to $\phi$ is due to the removal of the degeneracies of the energy eigenstates in the presence of impurity in the ring.

\section{Persistent Current: Rings with Fixed $N_{e}$}

In Fig. 6, we show the variation of the persistent currents for some one-channel disordered rings considering the ring size $N=50$ and the impurity strength $W=1$. Figure 6 (a) corresponds to the persistent currents for the rings described with odd number of electrons, while Fig. 6(b) represents the currents for the rings with even number of electrons. It is observed that the current varies continuously as a function of $\phi$ and gets much reduced amplitude compared to the results obtained in the impurity-free rings (see Fig. (3)). The 


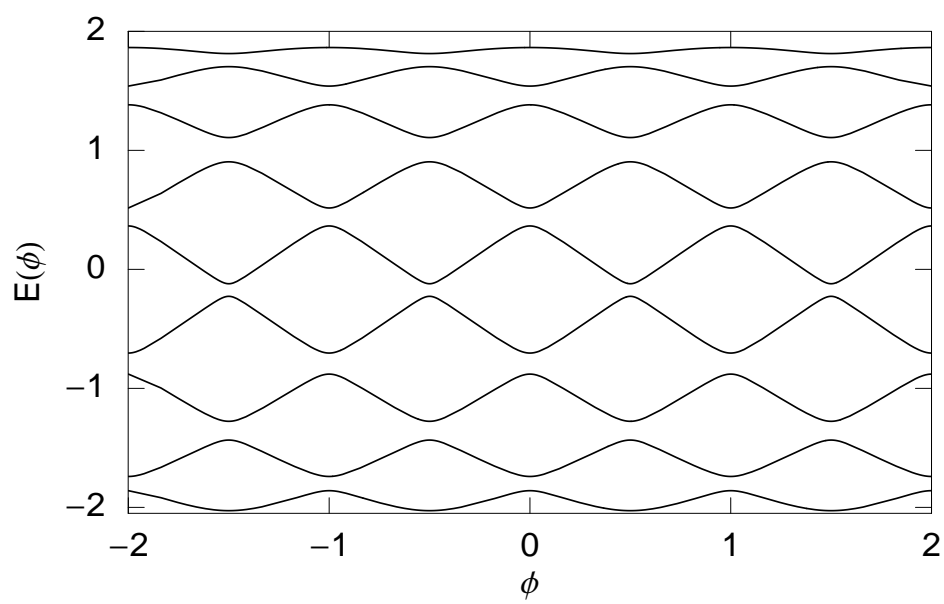

Figure 5: Electron energy levels as a function of the flux $\phi$ for a one-channel ring $(N=10)$ in the presence of impurity with strength $W=1$.

continuous variation of the current is clearly visible from the variation of the energy spectrum since they become continuous as long as the impurities are introduced in the ring. On the other hand, the suppression of the current amplitudes is due to the localization effect [42] of the energy eigenstates in the presence of impurity. Here all the results are described for some typical disordered configurations of the ring, and in fact we examine that the qualitative behavior of the persistent currents do not depend on the specific realization of the disordered configurations. This is the generic feature of persistent current for any one-channel non-interacting rings in the presence of impurity those are described with fixed number of electrons $N_{e}$.

\section{Persistent Current: Rings with Fixed $\mu$}

The behavior of the current-flux characteristics is quite interesting in the presence of impurity for the rings described with fixed chemical potential $\mu$, instead of $N_{e}$. As representative example, in Fig. 7 we plot the $I$ - $\phi$ characteristics for some one-channel disordered rings those are described with fixed $\mu$, where (a) represents the result for the ring with $\mu=-1$ and (b) denotes the result for the ring considering $\mu=-1.5$. From these results we can emphasize that depending on the choices of $\mu$ the current shows different behavior as a function of $\phi$ and in all such cases the current exhibits only $\phi_{0}$ periodicity.

\subsection{Non-Interacting Multi-Channel Systems}

Now we focus our study on the behavior of persistent currents in non-interacting multichannel systems [41]. A schematic representation of such a multi-channel system of cylindrical geometry threaded by a magnetic flux $\phi$ is given in Fig. 8. Considering the lattice 

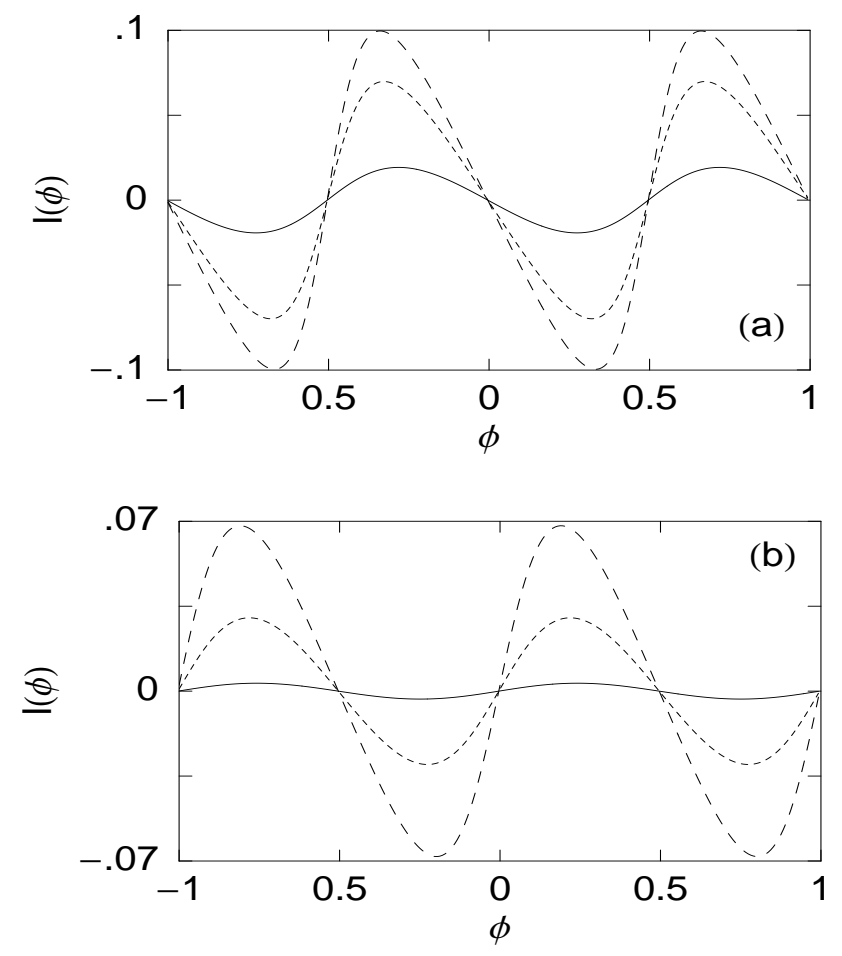

Figure 6: $I-\phi$ characteristics for the disordered $(W=1)$ one-channel rings $(N=50)$ with fixed number of electrons. The solid, dotted and dashed curves in (a) correspond to $N_{e}=9,15$ and 23 electrons respectively, while in (b) they correspond to $N_{e}=8,14$ and 22 electrons respectively.

spacing both in the longitudinal and transverse directions are identical i.e., the surface of the cylinder forms a square lattice, we can write the Hamiltonian of the system by the tight-binding formulation as,

$$
H=\sum_{x} \epsilon_{x} c_{x}^{\dagger} c_{x}+\sum_{<x x^{\prime}>}\left[t_{x x^{\prime}} e^{i \theta_{x x^{\prime}}} c_{x}^{\dagger} c_{x^{\prime}}+t_{x x^{\prime}} e^{-i \theta_{x x^{\prime}}} c_{x^{\prime}}^{\dagger} c_{x}\right]
$$

where $\epsilon_{x}$ is the site energy of the lattice point $x$ of coordinate, say, $(i, j) . t_{x x^{\prime}}$ is the hopping strength between the lattice points $x$ and $x^{\prime}$ and $\theta_{x x^{\prime}}$ is the phase factor acquired by the electron due to the longitudinal hopping in the presence of magnetic flux $\phi$. The study of persistent currents in such multi-channel systems becomes much more relevant compared to strictly one-dimensional rings (see Fig. (1), where we get only one channel that carries current, since most of the conventional experiments are performed in rings with finite width. Here we will describe the characteristic properties of persistent currents for some non-interacting multi-channel rings concerning the dependence of the current on total number of electrons $N_{e}$, chemical potential $\mu$, strength of disorder $W$ and number of channels. All the results studied here are performed only at absolute zero temperature. 

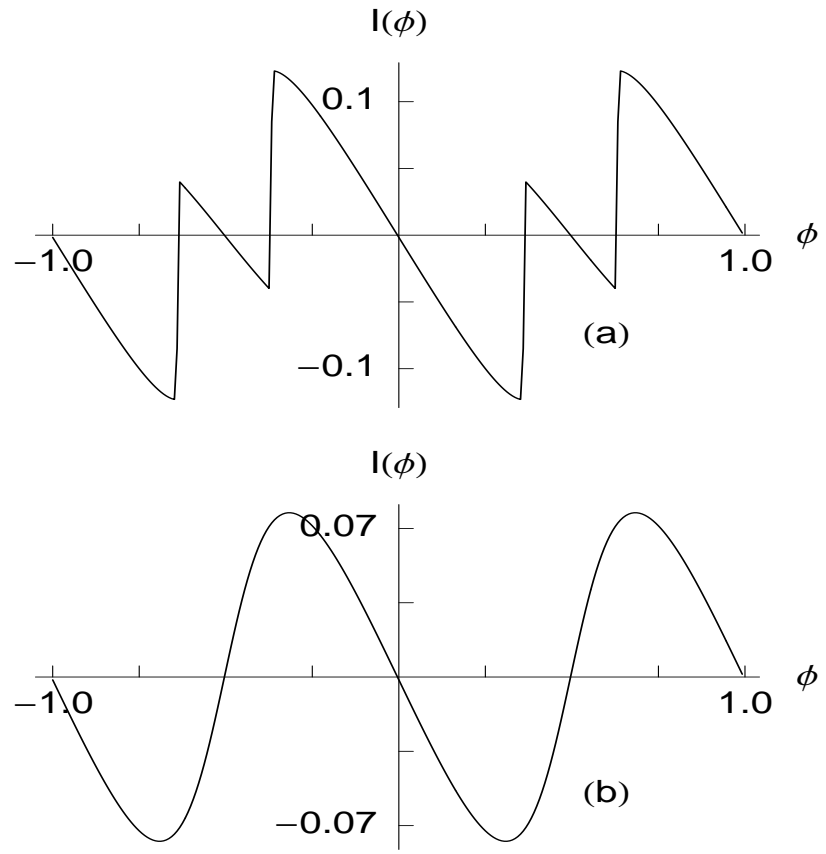

Figure 7: $I-\phi$ characteristics for the disordered $(W=1)$ one-channel rings $(N=50)$ described with constant $\mu$, where (a) $\mu=-1$ and (b) $\mu=-1.25$.

\subsubsection{Energy Spectra}

In order to present the behavior of persistent currents in mesoscopic multi-channel systems, let us first describe the energy-flux characteristics of a small cylindrical system that threads a magnetic flux $\phi$. To have a deeper insight to the problem we begin our discussion with the simplest possible system which can be calculated analytically. This is the case of a two-layer impurity free cylinder threaded by a magnetic flux $\phi$. This cylindrical system can be treated as two one-channel rings placed one above the other and they are connected by some vertical bonds (like as in Fig. 8). For strictly onedimensional ring i.e., for one layer the energy of $n$th eigenstate is expressed in the form $E_{n}(\phi)=2 t \cos \left[\frac{2 \pi}{N}\left(n+\frac{\phi}{\phi_{0}}\right)\right]$ (see Eq. [11), where $n=0, \pm 1, \pm 2, \ldots$. Here $t$ is the nearestneighbor hopping strength and $N$ is the total number of lattice points in the ring. The behavior of such energy levels as a function of flux $\phi$ is shown in Fig. 9(a), where we take $N=10$. From this figure it is observed that the energy levels are bounded within the range -2 to 2 in the scale of $t$ and the crossing of the energy levels (flux points where the energy levels have degeneracy) occurs at half-integer or integer multiples of $\phi_{0}$. Now as we add another one ring with the previous one-dimensional ring and connect it by $N$ vertical bonds it becomes a cylinder with two layers. For such a system, we get two different energy bands of discrete energy levels, each of which contains $N$ number of energy levels 


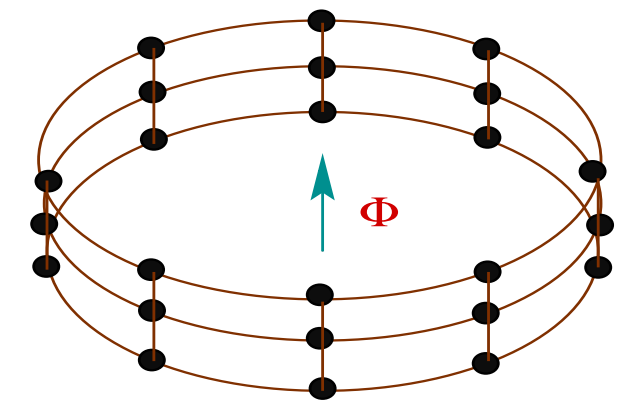

Figure 8: Schematic view of a multi-channel cylinder threaded by a magnetic flux $\phi$.

and they are respectively expressed in the form, $E_{1 n}(\phi)=t+2 t \cos \left[\frac{2 \pi}{N}\left(n+\frac{\phi}{\phi_{0}}\right)\right]$ and $E_{2 n}(\phi)=-t+2 t \cos \left[\frac{2 \pi}{N}\left(n+\frac{\phi}{\phi_{0}}\right)\right]$, where the symbol $n$ corresponds to the same meaning as above and $t$ is the nearest-neighbor hopping strength which is identical both for the longitudinal and transverse directions. In Fig. 9(b), we plot the energy levels of a small impurity-free cylinder considering $N=10$, where the solid and dotted curves correspond to the energy levels in the two separate energy bands respectively. These two different energy bands are bounded respectively in the range -1 to 3 and -3 to 1 in the scale of t. Accordingly, an overlap energy region appears for the two energy bands in the range -1 to 1 as shown in the figure (Fig. 9(b)). In this overlap region, the energy levels cross each other at several other flux points in addition to the half-integer and integer multiples of $\phi_{0}$ which provide different characteristic features of persistent currents. For cylinders with more than two layers, we get more energy bands like above and therefore several other overlap energy regions appear in energy spectra.

In the presence of impurity in multi-channel cylinders, gaps open at the crossing points of the energy levels and they become a continuous function of the flux $\phi$, like as in the one-channel disordered rings (Fig. 5). In all such perfect and disordered multi-channel cylinders, energy levels vary periodically with period $\phi_{0}$.

Now we shall describe the characteristic features of persistent currents for some small cylindrical systems, and our results might be quite helpful to explain the characteristic properties of persistent currents for larger system sizes.

\subsubsection{Persistent Current}

\section{Perfect Cylinders}

In this sub-section we study persistent currents of some impurity-free multi-channel systems of cylindrical geometry with two layers concerning the dependence of the current on total number of electrons $N_{e}$ and chemical potential $\mu$. As illustrative example, in Fig. 10 

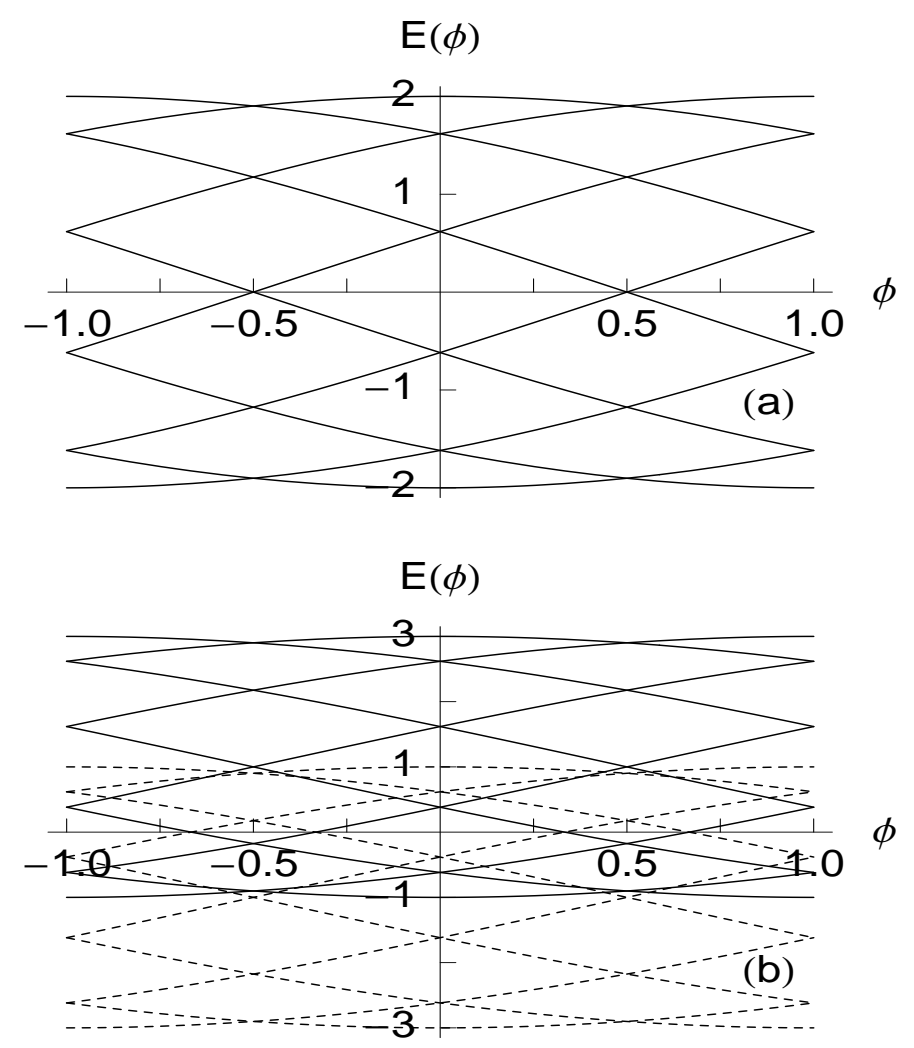

Figure 9: Energy levels as a function of the magnetic flux $\phi$, for (a) the perfect ring (one layer) with $N=10$ and (b) the perfect cylinder with two layers taking $N=10$ in each of these layers.

we plot the current-flux characteristics for some perfect cylinders considering $N=100$ in each of these two layers. Let us first describe the behavior of persistent currents for the cylinders those are described with fixed number of electrons. The first column of Fig. 10 corresponds to the currents for the systems with fixed $N_{e}$. To emphasize the effect of the energy overlap region on persistent currents here we study the systems for three different values of $N_{e}$. Figures 10(a) and (c) correspond to the currents for the systems with $N_{e}=25$ (low) and $N_{e}=185$ (high) respectively. The result for the intermediate value of $N_{e}\left(N_{e}=100\right)$ is shown in Fig. 10(b). It is shown that both for the low and high values of $N_{e}$, the persistent currents get saw-tooth like nature with sharp transitions only at half-integer multiple of $\phi_{0}$, similar to that of strictly one-channel impurity-free rings with odd $N_{e}$ (see Fig. 3(a)). This behavior can be explained as follow. For the system with $N_{e}=25$, the net persistent current is obtained by taking the sum of the lowest 25 energy eigenstates those lie below the energy overlap region. Now away from this overlap region, the energy levels behave exactly in the same way with strictly one-channel rings and therefore the current shows similar kind of saw-tooth shape as observed in one-channel 
rings. On the other hand, for the system with $N_{e}=185$ the situation is quite different than the previous one. To obtain the net current for this case we cross the overlap energy region since the highest energy level that contributes current lies far above of this overlap

I ( $\phi)$

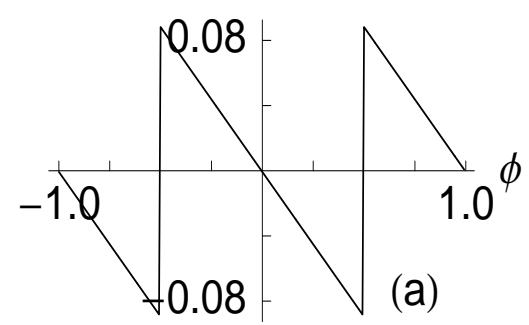

I ( $\phi)$

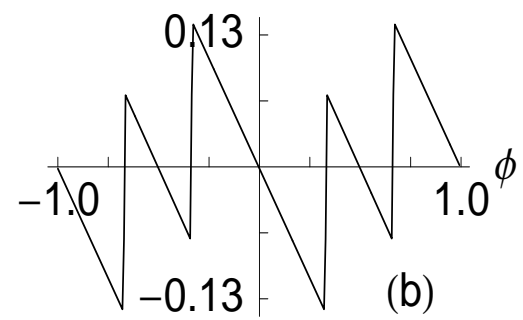

$I(\phi)$

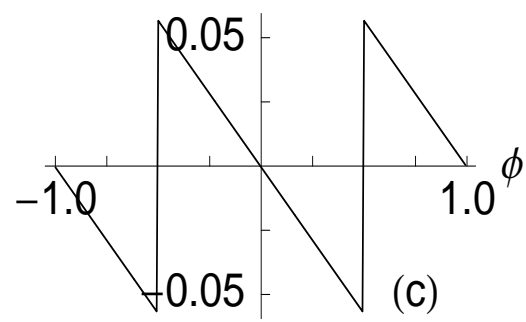

$\mathrm{I}(\phi)$

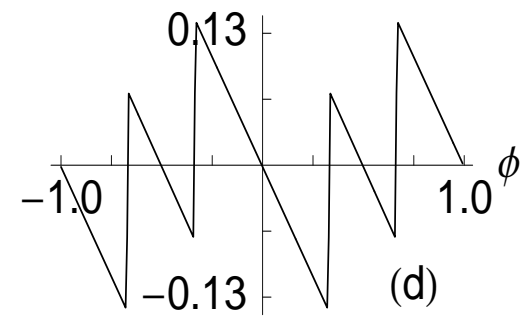

$\mathrm{I}(\phi)$

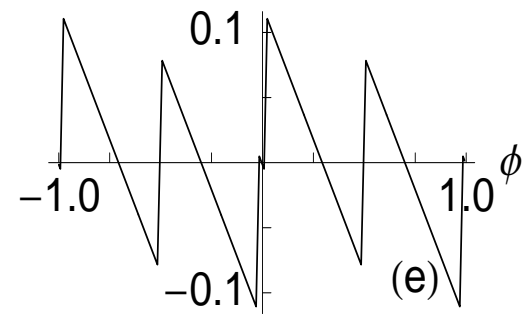

$\mathrm{I}(\phi)$

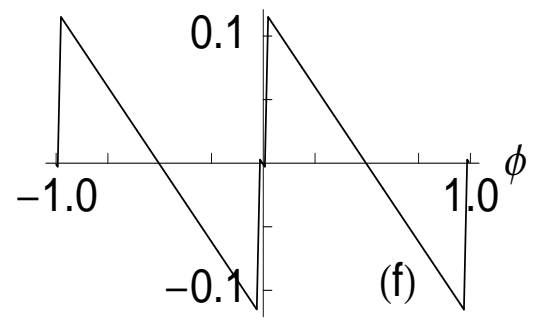

Figure 10: $I$ versus $\phi$ curves of the perfect cylinders with two layers taking $N=100$ in each layer. The results for the fixed $N_{e}$ are plotted in the first column, where (a) $N_{e}=25$, (b) $N_{e}=100$ and (c) $N_{e}=185$, while in the second column the results are plotted for the fixed $\mu$, where (d) $\mu=0$, (e) $\mu=-0.5$ and (f) $\mu=-1.5$.

region. The net contribution to the current from the energy levels within this overlap region vanishes and therefore no new feature appears in the persistent current compared to the system with $N_{e}=25$. Now we describe the result plotted in Fig. 10(b), where the system contains intermediate value of $N_{e}\left(N_{e}=100\right)$. For such a case the persistent current shows some additional kink-like structures across $\phi= \pm 0.5$. These kinks are due to the different contributions of the energy levels in the overlap energy region, since in the overlap region energy levels have more degeneracy at several other flux points rather than the half-integer and integer multiples of $\phi_{0}$. For other multi-channel systems with more 
than two layers, some more kinks may appear in persistent current at different values of $\phi$ depending on the choice of $N_{e}$ and the number of layers.

In multi-channel systems where we fix the chemical potential $\mu$ instead of the total number of electrons $N_{e}$, we also get different kink-like structures in the persistent currents as observed from the results plotted in the second column of Fig. 10. For all such systems described either with fixed $N_{e}$ or $\mu$, the current exhibits only $\phi_{0}$ flux-quantum periodicity.

\section{Dirty Cylinders}

To illustrate the effect of the impurity on persistent currents in multi-channel systems, here we concentrate our study in some dirty cylinders considering the same system size

$\mathrm{I}(\phi)$

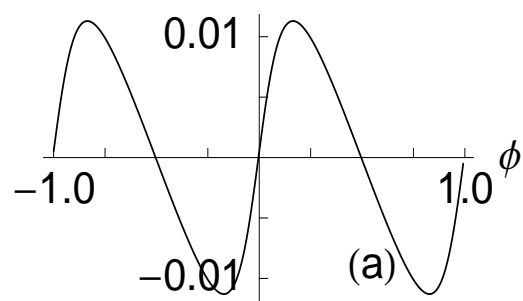

$\mathrm{I}(\phi)$

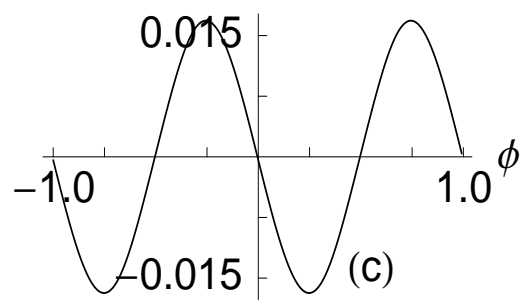

$\mathrm{I}(\phi)$

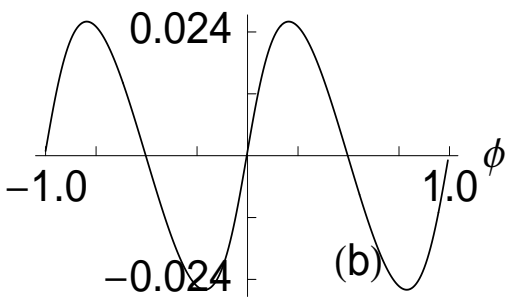

$\mathrm{I}(\phi)$

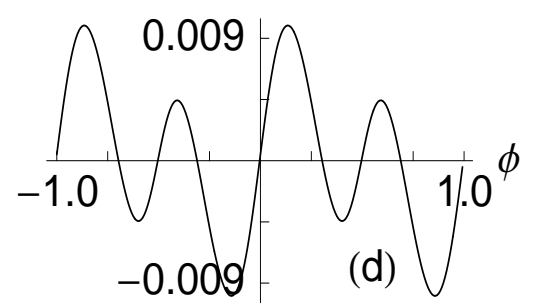

Figure 11: Current-flux characteristics for the disordered $(W=1)$ cylinders with two layers considering $N=100$ in each layer. The results for the fixed $N_{e}$ are plotted in the first column, where (a) $N_{e}=50$ and (b) $N_{e}=100$, while the results for the constant $\mu$ are plotted in the second column, where (c) $\mu=0$ and (d) $\mu=-0.5$.

as taken above for the perfect systems. In Fig. 11, we plot the current-flux characteristics for some disordered cylinders where the impurities are given only in the site energies $\left(\epsilon_{x}\right)$ by choosing them randomly from a "Box" distribution function of width $W=1$. All the results are plotted for isolated disordered configurations of the system. The first column of Fig. 11 represents the persistent currents for the cylinders described with fixed $N_{e}$, while the second column of this figure corresponds to the currents for the systems with fixed $\mu$. It is observed that, in all these cases the persistent current varies continuously as a function of flux $\phi$ showing $\phi_{0}$ periodicity. The explanation of such a continuous 
variation has already been described in our previous studies. Another significant point observed from Figs. 11(a) and (b) is that, the slope of the current in the zero-field limit $(\phi \rightarrow 0)$ changes in opposite direction though both for the two cases the systems contain even number of electrons. This provides the signature of random sign of low-field currents in multi-channel systems. The detailed description of low-field magnetic response will be available in the forthcoming section (Section 3).

Thus we can emphasize that the behavior of persistent current in multi-channel systems strongly depends on the disordered configurations, total number of electrons $N_{e}$, chemical potential $\mu$ and also on the total number of channels.

\section{Low-Field Magnetic Response on Persistent Cur- rent}

The diamagnetic or the paramagnetic sign of low-field persistent currents also becomes a controversial issue due to discrepancy between theory and experiment. From the theoretical calculations, Cheung et al. [13] predicted that the sign of persistent current is random depending on the total number of electrons, $N_{e}$, in the system and on the specific realization of the disordered configurations of the ring. Both the diamagnetic and the paramagnetic responses were also observed theoretically in mesoscopic Hubbard rings by $\mathrm{Yu}$ and Fowler [43]. They showed that the rings with odd $N_{e}$ exhibit the paramagnetic response, while those with even $N_{e}$ have the diamagnetic response in the limit $\phi \rightarrow 0$. In other recent work Waintal et al. [44] have also discussed theoretically the sign of persistent current of $N$ electrons in one-dimensional rings and provided several interesting results. In an experiment on $10^{7}$ isolated mesoscopic $\mathrm{Cu}$ rings, Levy et al. [4] had reported the diamagnetic response for the low-field currents, while with Ag rings Chandrasekhar et al. 6] got the paramagnetic response. In a recent experiment, Jariwala et al. [7] have got the diamagnetic persistent currents with both integer and half-integer flux-quantum periodicities in an array of 30-diffusive mesoscopic gold rings. The diamagnetic sign of the currents in the vicinity of zero magnetic field were also found in an experiment [8] on $10^{5}$ disconnected Ag ring. The sign of the low-field current is a priori not consistent with the theoretical predictions. In this section, we will study the nature of the low-field magnetic susceptibility of mesoscopic rings and cylinders through some exact calculations.

The magnetic susceptibility of any mesoscopic ring/cylinder can be obtained from the general expression [41,

$$
\chi(\phi)=\frac{N^{3}}{16 \pi^{2}}\left[\frac{\partial I(\phi)}{\partial \phi}\right]
$$


Calculating the sign of $\chi(\phi)$, one can predict whether the current is paramagnetic or diamagnetic. Here we focus our attention on the systems either with fixed number of electrons $\left(N_{e}\right)$ or with fixed chemical potential $(\mu)$.

\subsection{One-Channel Mesoscopic Rings}

Let us first study the low-field magnetic susceptibility of impurity-free one-channel mesoscopic rings with fixed $N_{e}$. Figure 12(a) shows the variation of $\chi(\phi)$ as a function of $N_{e}$
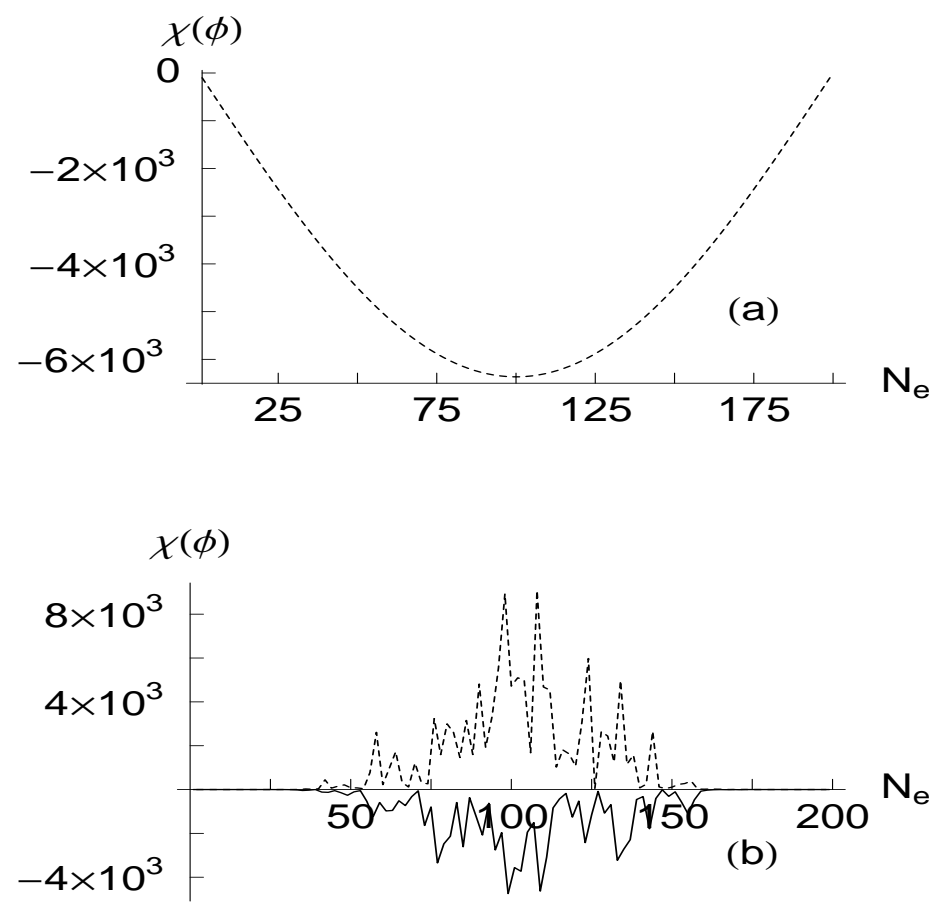

Figure 12: Low-field magnetic susceptibility as a function of $N_{e}$, for (a) perfect rings $(W=0)$ and (b) rings in the presence of impurity $(W=1)$ with $N=200$. The solid and dotted lines in (b) correspond to the rings with odd and even $N_{e}$ respectively.

for a perfect ring with $N=200$ in the limit $\phi \rightarrow 0$. It is noticed that, both for the even and odd $N_{e}$ the current has only the diamagnetic sign. This diamagnetic sign of the low-field currents can be easily understood from the slope of the current-flux characteristics of the one-channel impurity-free rings (see the curves plotted in Fig. (3)). From these curves it follows that the persistent current always exhibits negative slope at low-fields. Therefore, it can be predicted that for perfect one-channel rings the current shows only the diamagnetic sign near zero-field limit, irrespective of the total number of electrons $N_{e}$ i.e., whether the rings contain odd or even $N_{e}$. 
The effects of disorder on the low-field currents are quite interesting, and our results illustrate that the sign of the currents, even in the presence of disorder, can be mentioned without any ambiguity both for the rings with odd and even $N_{e}$. In Fig. 12(b), we plot $\chi(\phi)$ as a function of $N_{e}$ for the disordered rings. Here we take $N=200$ and $W=1$. The solid and dotted lines in Fig. 12(b) correspond to the results for the rings with odd and even $N_{e}$ respectively. These curves show that the rings with odd $N_{e}$ exhibit only the diamagnetic sign for the low-field currents, while for even $N_{e}$ the low-field currents always have the paramagnetic sign. Physically, the disorder lifts all the degeneracies of the energy levels those were observed in a perfect ring, and as a result the sharp discontinuities of the $I-\phi$ characteristics (see the curves of Fig. 3) disappear. It may be noted that the slopes of the $I$ - $\phi$ curves for even and odd $N_{e}$ always have opposite signs near zero magnetic field (see the curves of Fig. (6). Thus for the one-dimensional disordered rings with fixed number of electrons, the sign of the low-field current is independent of the specific realization of the disordered configurations and depends only on the oddness or evenness of $N_{e}$.

\subsubsection{Effect of Temperature}

At finite temperature, we notice an interesting behavior of the low-field magnetic susceptibility of mesoscopic rings. Let us confine ourselves to the systems with even number of

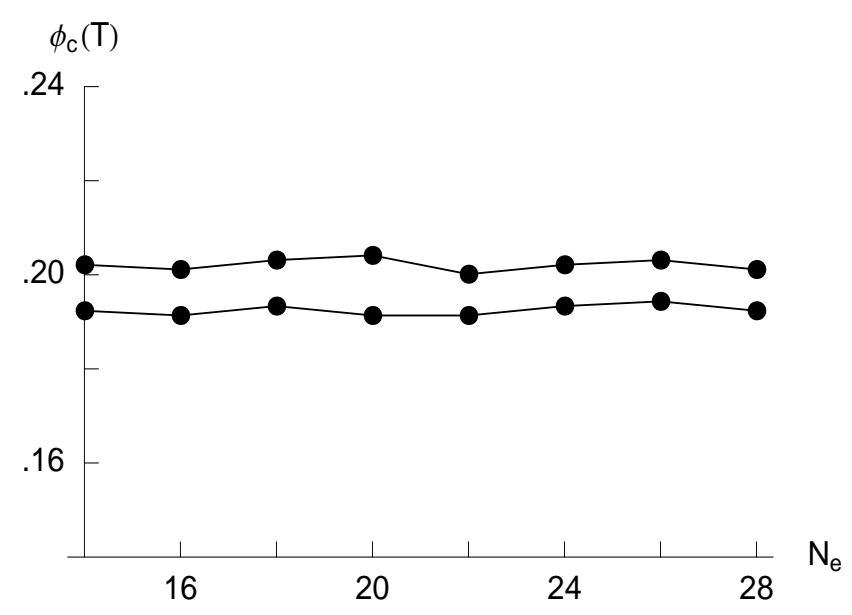

Figure 13: $\phi_{c}(T)$ versus $N_{e}\left(N_{e}=\right.$ even $)$ curves for perfect rings with size $N=45$.

electrons. At any finite temperature, the magnetic response of these systems are always paramagnetic both for the perfect and the dirty rings in the zero field limit. For a given system, this paramagnetism is observed over a certain range of $\phi$ close to $\phi=0$, say, in the domain $-\phi_{0} / 4 \leq \phi \leq \phi_{0} / 4$. Quite interestingly we observe that, at finite temperature the magnetic response of this particular system becomes diamagnetic beyond a critical field $\phi_{c}(T)$, even though $\left|\phi_{c}(T)\right|<\phi_{0} / 4$. 
In Fig. 13, we show the variation of the critical field $\phi_{c}(T)$ with respect to only even $N_{e}$ for a perfect one-channel ring of size $N=45$. The curve with higher values of $\phi_{c}(T)$ corresponds to the temperature $T / T^{\star}=1.0$, while the other curve corresponds to $T / T^{\star}=0.5$. Figure 14 represents the behavior of $\phi_{c}(T)$ for a dirty sample (with $W=1$ ) at the same two temperatures $T / T^{\star}=1.0$ (upper curve) and $T / T^{\star}=0.5$ (lower curve). From Figs. 13 and 14 it is clear that the critical value of $\phi$, where the transition

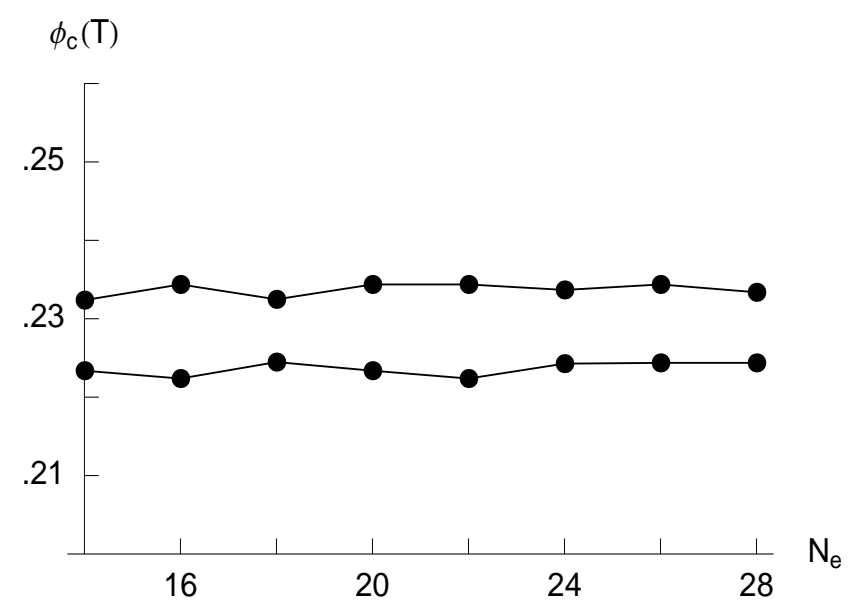

Figure 14: $\phi_{c}(T)$ versus $N_{e}\left(N_{e}=\right.$ even $)$ curves for dirty $(W=1)$ rings with size $N=40$.

from the paramagnetic to the diamagnetic phase takes place, increases with the increase of the temperature. Thus we see that, both for the perfect and dirty rings with even number of electrons there exists a critical value of magnetic flux $\phi_{c}(T)$, beyond which the magnetic response of the low-field currents exhibits a transition from the paramagnetic to the diamagnetic phase.

The situation is quite different even at zero temperature when we describe the system by constant chemical potential instead of fixed $N_{e}$. It may be noted that, only for some particular values of $\mu$ the system will have a fixed number of electrons, and for these values of $\mu$ the sign of the low-field currents can be predicted according to the above prescriptions. While, for all other choices of $\mu$ the total number of electrons varies even for a slight change in magnetic flux $\phi$ in the neighborhood of zero flux. Hence, it is not possible to predict the sign of the low-field currents precisely, even in the absence of any impurity in the system. Thus the sign of the low-field currents strongly depends on the choice of $\mu$, the strength of disorder and the choice of the disordered configurations.

\subsection{Multi-Channel Mesoscopic Cylinders}

We have also studied the low-field magnetic response for the mesoscopic rings of finite width [41]. Our study reveals that, for such systems it is not possible to predict the sign 
of the low-field currents precisely even for the impurity-free cases with fixed number of electrons. So we can conclude that, in the diffusive multi-channel mesoscopic rings the sign of the low-field currents is a highly unpredictable quantity as it can be easily affected by the total number of electrons $N_{e}$, chemical potential $\mu$, magnetic flux $\phi$, strength of disorder $W$, realizations of disordered configurations, etc. This is exactly the same picture what has been observed experimentally regarding the sign of the low-field currents.

\section{Concluding Remarks}

In this dissertation, we have demonstrated the behavior of the persistent current and low-field magnetic response in mesoscopic one-channel rings and multi-channel cylinders. All the results have been computed in the non-interacting electron picture withing the tight-binding framework. These results may be quite helpful for the beginners to realize the basic features of persistent current in metallic loops.

The characteristic properties of persistent current in the non-interacting one-channel rings and multi-channel cylinders have been presented in Section 2 showing its dependence on the total number of electrons $N_{e}$, chemical potential $\mu$, randomness and the total number of channels. All the calculations have been performed only at absolute zero temperature. In perfect one-channel rings with fixed $N_{e}$, persistent current shows sawtooth like behavior as a function of magnetic flux $\phi$ with sharp discontinuities at $\phi=$ $\pm n \phi_{0} / 2$ or $\pm n \phi_{0}$ depending on whether the system has odd or even $N_{e}$. On the other hand, some additional kinks may appear in the currents for the one-channel perfect rings with fixed $\mu$. The situation is quite different for the multi-channel perfect cylinders. In such cylindrical rings, the kinks appear in the persistent currents for both the cases with fixed $N_{e}$ or fixed $\mu$.

The diamagnetic or the paramagnetic sign of the low-field currents is a controversial issue due to the discrepancy between theory and experiment. In Section 3, we have examined the behavior of the low-field magnetic response of persistent currents by calculating the magnetic susceptibility in the limit $\phi \rightarrow 0$. In perfect one-channel rings, the low-field current exhibits only the diamagnetic sign irrespective of the parity of the total number of electrons $N_{e}$ i.e, whether $N_{e}$ is odd or even, while in the disordered rings currents have the diamagnetic or the paramagnetic nature depending on whether the rings contain odd or even $N_{e}$. The important point is that, for the disordered one-channel rings with fixed $N_{e}$ the sign of the low-field currents is completely independent of the specific realization of the disordered configurations. In this context we have also studied the effect of finite temperature and observed that both for the perfect and the dirty rings with even number 
of electrons, there exits a critical value of magnetic flux $\phi_{c}(T)$ beyond which the magnetic response of the low-field currents makes a transition from the paramagnetic to the

diamagnetic phase. But in dirty rings with constant chemical potential $\mu$, the sign of the low-field currents cannot be predicted since it strongly depends on the choices of $\mu$. Finally, in the case of multi-channel systems we have noticed that the sign of these currents cannot be predicted exactly, even in the perfect systems with fixed $N_{e}$ as it strongly depends on the choice of $N_{e}, \mu$, number of channels, disordered configurations, etc.

\section{References}

[1] Y. Aharonov and D. Bohm, Phys. Rev. 115, 485 (1959).

[2] F. Hund, Ann. Phys. (Leipzig) 32, 102 (1938).

[3] M. Büttiker, Y. Imry and R. Landauer, Phys. Lett. A 96, 365 (1983).

[4] L. P. Levy, G. Dolan, J. Dunsmuir and H Bouchiat, Phys. Rev. Lett. 64, 2074 (1990).

[5] D. Mailly, C. Chapelier and A. Benoit, Phys. Rev. Lett. 70, 2020 (1993).

[6] V. Chandrasekhar, R. A. Webb, M. J. Brady, M. B. Ketchen, W. J. Gallagher and A. Kleinsasser, Phys. Rev. Lett. 67, 3578 (1991).

[7] E. M. Q. Jariwala, P. Mohanty, M. B. Ketchen and R. A. Webb, Phys. Rev. Lett. 86, 1594 (2001).

[8] R. Deblock, R. Bel, B. Reulet, H. Bouchiat and D. Mailly, Phys. Rev. Lett. 89, $206803(2002)$.

[9] B. Reulet, M. Ramin, H. Bouchiat and D. Mailly, Phys. Rev. Lett. 75, 124 (1995).

[10] W. Rabaud, L. Saminadayar, D. Mailly, K. Hasselbach, A. Benoit and B. Etienne, Phys. Rev. Lett. 86, 3124 (2001).

[11] M. Büttiker, Phys. Rev. B 32, 1846 (1985).

[12] H-F Cheung, E. K. Riedel and Y. Gefen, Phys. Rev. Lett. 62, 587 (1989).

[13] H. F. Cheung, Y. Gefen, E. K. Riedel and W. H. Shih, Phys. Rev. B 37, 6050 (1988).

[14] R. Landauer and M. Büttiker, Phys. Rev. Lett. 54, 2049 (1985).

[15] N. Byers and C. N. Yang, Phys. Rev. Lett. 7, 46 (1961). 
[16] F. von Oppen and E. K. Riedel, Phys. Rev. Lett. 66, 84 (1991).

[17] G. Montambaux, H. Bouchiat, D. Sigeti and R. Friesner, Phys. Rev. B 42, 7647 (1990).

[18] H. Bouchiat and G. Montambaux, J. Phys. (Paris) 50, 2695 (1989).

[19] S. K. Maiti, J. Chowdhury and S. N. Karmakar, Phys. Lett. A 332, 497 (2004).

[20] B. L. Altshuler, Y. Gefen and Y. Imry, Phys. Rev. Lett. 66, 88 (1991).

[21] A. Schmid, Phys. Rev. Lett. 66, 80 (1991).

[22] M. Abraham and R. Berkovits, Phys. Rev. Lett. 70, 1509 (1993).

[23] A. Müller-Groeling and H. A. Weidenmuller, Phys. Rev. B 49, 4752 (1994).

[24] I. O. Kulik, Physica B 284, 1880 (2000).

[25] H. B. Chen and J. W. Ding, Physica B 403, 2015 (2008).

[26] P. A. Orellana, M. L. Ladron de Guevara, M. Pacheco and A. Latge, Phys. Rev. B 68, 195321 (2003).

[27] I. O. Kulik, JETP Lett. 11, 275 (1970).

[28] S. K. Maiti, J. Comput. Thoer. Nanosci. 5, 2135 (2008).

[29] S. K. Maiti, Fractional periodic persistent current in a twisted normal metal loop: an exact result, J. Comput. Thoer. Nanosci. (in press).

[30] S. K. Maiti and F. Aeenehvand, Persistent current in a mesoscopic cylinder: effects of transverse magnetic field, J. Comput. Thoer. Nanosci. (in press).

[31] S. K. Maiti, Int. J. Mod. Phys. B 22, 4951 (2008).

[32] T. Giamarchi and B. S. Shastry, Phys. Rev. B 51, 10915 (1995).

[33] S. K. Maiti, J. Chowdhury and S. N. Karmakar, Solid State Commun. 135, 278 (2005).

[34] S. K. Maiti, J. Chowdhury and S. N. Karmakar, Synthetic Metals 155, 430 (2005).

[35] S. K. Maiti, IJMPB 21, 179 (2007). 
[36] S. K. Maiti, J. Chowdhury and S. N. Karmakar, J. Phys.: Condens Matter 18, 5349 (2006).

[37] K. Yakubo, Y. Avishai and D. Cohen, Phys. Rev. B 67, 125319 (2003).

[38] E. H. M. Ferreira, M. C. Nemes, M. D. Sampaio and H. A. Weidenmüller, Phys. Lett. A 333, 146 (2004).

[39] S. K. Maiti, IJMPB 21, 3001 (2007); [Addendum: IJMPB 22, 2197 (2008)].

[40] S. K. Maiti, Phy. Scr. 73, 519 (2006); [Addendum: Phy. Scr. 78, 019801 (2008)].

[41] S. K. Maiti, Physica E 31, 117 (2006).

[42] P. A. Lee and T. V. Ramakrishnan, Rev. Mod. Phys. 57, 287 (1985).

[43] N. Yu and M. Fowler, Phys. Rev. B 45, 11795 (1992).

[44] X. Waintal, G. Fleury, K. Kazymyrenko, M. Houzet, P. Schmitteckert and D. Weinmann, Phys. Rev. Lett. 101, 106804 (2008). 